\title{
Correction to: Complex Adaptive Systems and Interactive Granular Computing
}

Andrzej Skowron

\section{Correction to:}

Chapter "Complex Adaptive Systems and Interactive

Granular Computing" in: K. Saeed and W. Homenda (Eds.):

Computer Information Systems and Industrial Management, LNCS 9842, https://doi.org/10.1007/978-3-319-45378-1_2

The acknowledgement section of this paper originally referred to grant DEC-2013/09/B/ST6/01568. The reference to this grant has been removed from the acknowledgement section at the request of one of the authors. 